\title{
Artifacts removal from breath sound recordings in pediatric population
}

\author{
Yusuf A Amrulloh ${ }^{1, *}$, Jawahir A K Haq ${ }^{2}$ \\ ${ }^{1}$ Electrical Engineering Department, Universitas Islam Indonesia, Yogyakarta, Indonesia \\ ${ }^{2}$ B2TKS-BPPT, Kawasan Puspiptek Gedung 220, Tangerang Selatan, Indonesia
}

\begin{abstract}
Breath sound recordings from pediatric subjects pose more processing complications. Children, especially the younger ones, are not able to follow instructions to stay calm during recording. This makes their recordings not only contain stationary artifacts but also non-stationary artifacts such as movement of subjects and their heartbeats. Further, the breath sounds from pediatric subjects also have lower magnitude compared to adults. In this work, we proposed to address those problems by developing a method to remove the artifacts from breath sound recordings. We implemented a combination of a Butterworth band pass filter and a discrete wavelet filter. We tested three types of wavelets (Coiflet, Symlet and Daubechies). Ten level decompositions and a set of hard thresholds were implemented in our work. Our results show that our developed method was capable of removing the artifacts significantly while maintaining the signal of interest. The highest signal to noise ratio improvement $(10.65 \mathrm{~dB})$ was achieved by 32 orders Symlet.
\end{abstract}

\section{Introduction}

Breath sound analysis is extremely important to support the initial diagnosis of respiratory tract infections [1]. It is used during physical examination at the first time patients admitted to hospitals or community health centres. In pediatric pneumonia, physicians use stethoscope to listen the breath sounds and to identify abnormal sounds such as crackle [2, 3], wheeze [4] or stridor [5].

Currently, electronics stethoscope is get used widely by physicians. In contrast with conventional stethoscope, the device is not only able to listen the breath sounds but also to record them for further analysis. Breath sound analysis of respiratory diseases in pediatric population is far different from adults due to the pathology and physiology. Dangerous diseases such as pneumonia mostly infect younger children less than five years [5]. Children in this age ranges are not able to follow instructions during physical examination. They tend to move and to cry. Further, their respiratory system is dissimilar with adults [6]. They have smaller lungs and airways that make the breath sounds magnitude weaker. The interference from their heart sound is higher because the number of heartbeat in younger children is much larger. All those artifacts impose the breath sounds and reduce their quality.

In this paper, we propose a method to remove the artifacts from breath sounds recorded in children with pneumonia. The novelties of this work are the subjects as well as the signal used in this study. Rather than involving adult subjects who are able to follow instruction to minimize artifacts during recording [7], we used actual breath sound recorded from from children with its natural artifacts. In facts, it is difficult to get a reference of artifact signals such as heartbeats during realtime recording. Therefore, a method for removing artifacts in the absence of reference signals is required.

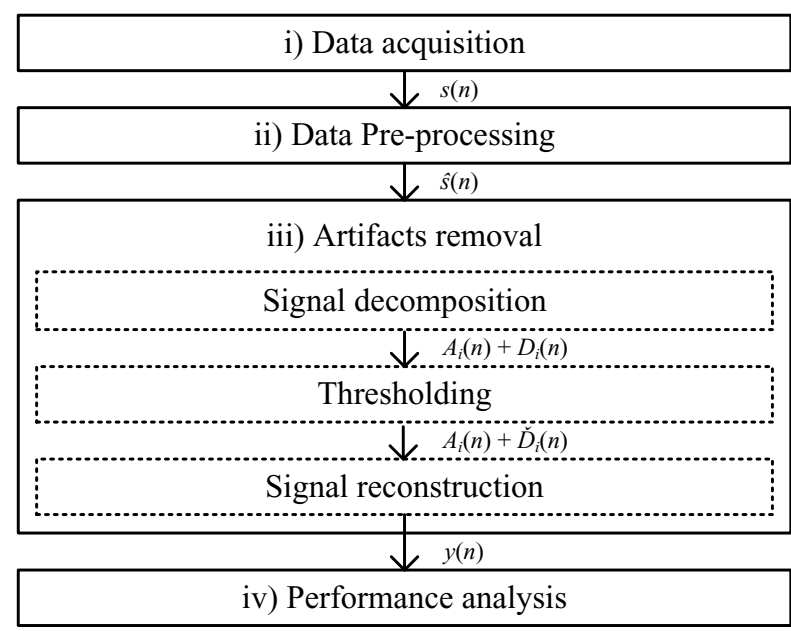

Fig. 1. Block diagram of the proposed method. It comprised of four main processes: i) data acquisition, ii) pre-processing, iii) artifacts removal and iv) performance analysis of the proposed method.

The work in presented in this paper consisted of four processes as shown in Fig 1. It comprised of i) data acquisition, ii) Pre-processing using band pass filter, iii) artifact removal using discrete wavelet transform filter, and iv) performance analysis on different types of

\footnotetext{
* Corresponding author: yusuf.amrulloh@uii.ac.id
} 
wavelet. Details of the processes are described in the following sub sections.

\section{Methods}

\subsection{Data Acquisition}

We recorded the breath sounds from pediatric subjects diagnosed as pneumonia in the pediatric ward of PDHI Islamic Hospital Yogyakarta Indonesia. To record the breath sounds, we used a Thinklab One Digital Stethoscope (Thinklabs USA). We connected the electronic stethoscope to an Android mobile phone and a head set via Thinklink connector. TapeMachine Lite Recorder application was used to record the breath sounds. The connection of the hardware used for recording is illustrated in Fig 2.

The recording was started after informed consent had been completed. We apply the electronic stethoscope on the chest wall of the subjects to record the breath sounds. The sampling rate was set at $44.1 \mathrm{kHz}, 16$ bit to maintain the recording quality. The recorded breath sounds were saved in wav format files in mobile phone's memory. Once the recording had completed, we transferred the files to a computer for further processing.

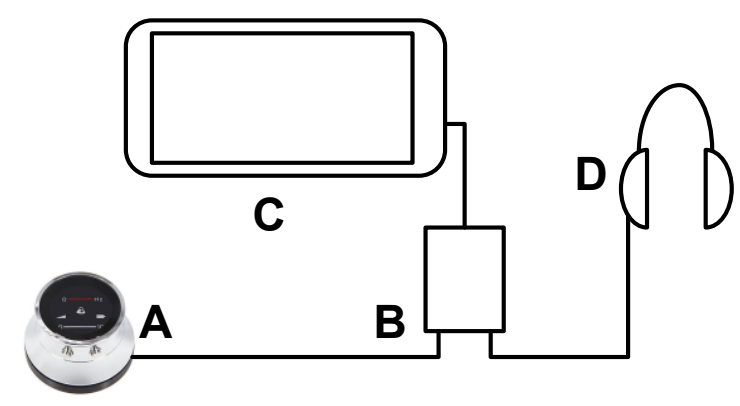

Fig. 2. Hardware used in this study. It comprised of A) Thinklab One Digital Stethoscope, B) Think Link connector, C) Android mobile phone and D) Headset for

\subsection{Pre-processing}

The pre-processing was carried to reduce the stationary artifacts. Let $s(n)$ denotes the breath sound recordings. After we had obtained the breath sounds data, then we preprocessed them to reduce the ambient noise and electronic noise having spectrum below $50 \mathrm{~Hz}$ and higher than 2000 Hz. We implemented a ten-order Butterworth band pass filter having cut off frequency of $50 \mathrm{~Hz}$ and $2000 \mathrm{~Hz}$. The cut off frequencies were designed based on the noise profile of the recordings. The output signal from the preprocessing is denoted as $\hat{S}(n)$.

\subsection{Artifacts removal}

In this process, the output signal from the pre-processing $\hat{s}(n)$ was processed to remove the non-stationary artifacts.
We developed a wavelet filter based-system for this purpose. Our system comprised of three stages:

\subsubsection{Decomposing sound signal using a discrete wavelet transform filter}

In this stage we decompose signal $\hat{s}(n)$ using discrete wavelet transform filter (DWT). Let $i(i=1,2, \ldots N)$ denotes the decomposition level. Passing $\hat{s}(n)$ through $N$ level DWT generates approximation coefficient $A_{i}$ and details coefficient $D_{i}$ in each level as given in Eq 1 and Eq 2 [8].

$$
\begin{aligned}
& A_{i}[n]=\sum_{k=-\infty}^{\infty} \hat{S}[n] g[2 n-k] \\
& D_{i}[n]=\sum_{k=-\infty}^{\infty} \hat{s}[n] h[2 n-k]
\end{aligned}
$$

where $\left(A_{i}\right)$ and $\left(D_{i}\right)$ are the approximation and details of the low and high frequency components, respectively. The coefficients were generated from the convolution of input signal $\hat{s}(n)$ with impulse response of high pass filter (HPF) $g$ and low pass filter (LPF) $h$ in each level of decomposition $i$. The term $2 n$ means that the output of HPF and LPF were down sampled by 2 . This means sound signal in each level will have half bandwidth from the previous level.

In this work, we set the maximum decomposition level $N=10$ to achieve the minimum frequency resolution below to $21 \mathrm{~Hz}$. We tested three types of wavelets including Coiflet wavelet (coifl5), Symlet wavelet (sym5, sym 8, sym 20, sym 32$)$, and Daubechies wavelet ( $d b 5, d b 8$, $d b 20, d b 32)$. After decomposing the signal, we passed the signal to thresholding process described in the following sub-section.

\subsubsection{Comparing the decomposed signals with a set of thresholds}

To eliminate the artifacts, in this process we compared the details coefficients in each level $D_{i}$ with a set of thresholds. Coefficients with absolute values higher than the defined threshold $\tau$ were set to zero while coefficients below the threshold were kept. Threshold function is given in Eq 3.

$$
D_{i}= \begin{cases}0, & \left|D_{i}\right| \geq \tau \\ D_{i}, & \left|D_{i}\right|<\tau\end{cases}
$$

where $\check{D}_{i}$ is the output of thresholding in i decomposition level. In this process, artifacts were assumed as the decomposed signal having absolute value higher than the threshold values. Therefore, the artifacts magnitudes were reduced to zero.

\subsubsection{Reconstructing signals using inverse discrete wavelet transform}

In this stage, we reconstructed the entire decomposed signal $\left(A_{i}\right.$ and $\left.\check{D}_{i}\right)$ from the thresholding process. Approximation coefficients $A_{i}$ and details coefficients $\check{D}_{i}$ 
were up sampled by two and then processed through high pass filter and low pass filter, respectively. The filters were used to remove the aliased signal from previous process. Output signal from this process was the summation of the output signal from HPF and LPF denoted as $y(n)$, the breath sounds that free from the artifacts.

\subsubsection{Performance evaluation if the methods}

To evaluate the performance of our developed method, we compute the signal to noise ratio (SNR in decibel/dB) of the input signal $s(n)$ and output signal $y(n)$. The SNR is given in Eq. 4.

$$
S N R_{j}=10 \log 10\left(\sum_{n=0}^{m} \frac{|\xi(n)|^{2}}{|\psi(n)|^{2}}\right)
$$

where $\xi$ is the breath sound events, $\psi$ is the artifacts and noise while $m$ is the length of data used for computation. The term $j$ represents the SNR for input signal $(j=1)$ and SNR for output signal from wavelet filter $(j=2)$. Then we computed the difference between input and output SNR $(\triangle \mathrm{SNR})$ as given in Eq 5.

$$
\Delta S N R=S N R_{j=2}-S N R_{j=1}
$$

The best performance of the wavelet filter was indicated by the highest $\triangle \mathrm{SNR}$.

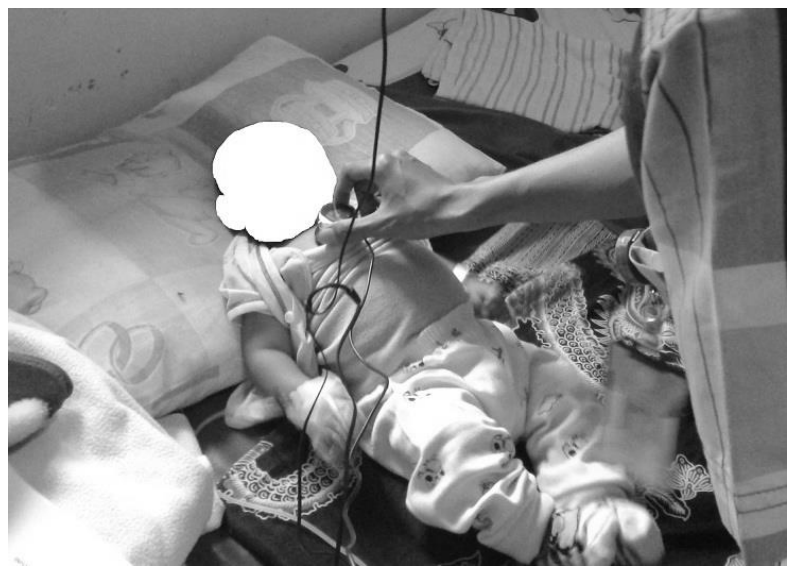

Fig. 3. Illustration of an auscultation process in a pediatric subject.

\section{Results and discussion}

\subsection{Data acquisition}

We recorded breath sounds from eleven (9 males and 2 female) pediatric subjects diagnosed as pneumonia. The age range was from two months to fifteen months with average of eight months. In the physical examination, there were symptoms such as fever ( 5 subjects), cold (5 subjects), and breathing difficulty ( 8 subjects). Cough was found in all of subjects. The auscultation process is illustrated in Fig 3.

Of the eleven subjects, we only able to use nine subjects (subject no 3 to 11) due to clipping problems in the two other subjects (subject no 1 and 2). Our working data consisted of 7 male and 2 female subjects. The average of recording duration was 118 second (minimum duration was 65 seconds and maximum duration 166 seconds). The duration of recording depended on the condition of the subjects.

In Fig 4 we illustrate the example of artifacts from rubbing sounds and heart sounds. It can be seen that artifacts contain frequency components lower than $50 \mathrm{~Hz}$ to $550 \mathrm{~Hz}$ wherein the spectrum of breath sound also exist. The artifacts may have duration from 0.05 seconds up to 0.2 seconds. This shows that artifacts in the recordings may overlap with breath sounds in both time domain as well as frequency domain.

The typical breathing sound obtained from the auscultation from pediatric subjects shown in Fig 5(A). It can be seen that stationary and non-stationary artifacts imposed the breath sound signal. The spectrum of the signal is shown in Fig 6(A). We processed the signal through pre-processing and artifact removal using discrete wavelet transform filter.

\subsection{Pre-processing}

Our frequency of interest was from $50 \mathrm{~Hz}$ to $2 \mathrm{kHz}$. Therefore, we developed ten order Butterworth band pass filter having low and high cut off frequency of $f_{l}=50 \mathrm{~Hz}$ and $f_{h}=2 \mathrm{kHz}$, respectively. This filter was designed to reduce the stationary artifacts. The response of the developed filter is as follows. The attenuation in $f_{l}$ is $18,81 \mathrm{~dB}$ and $7.62 \mathrm{~dB}$ in $f_{h}$. The attenuation in the first octave in $f_{l}$ and $f_{h}$ is $79,3 \mathrm{~dB}$ and $63.4 \mathrm{~dB}$, respectively.
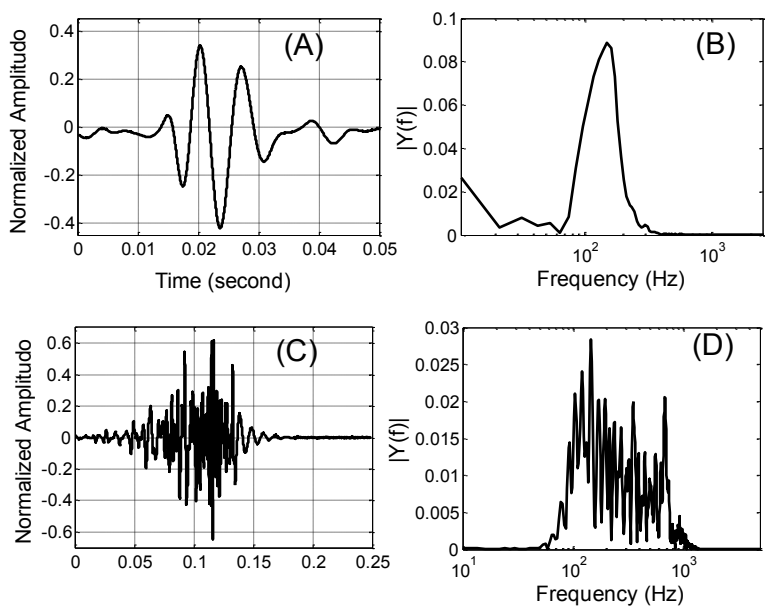

Fig. 4. Illustration of artifacts from heart sound (A) and rubbing sound (B). The spectrum of heart sound and rubbing sounds are illustrated in (C) and (D) respectively.

We illustrated the output of the pre-processing in Fig 5(B). It can be seen that the filter was able to reduce the low frequency noise. The spectrum of the signal after filtering is shown in Fig 6(B). 


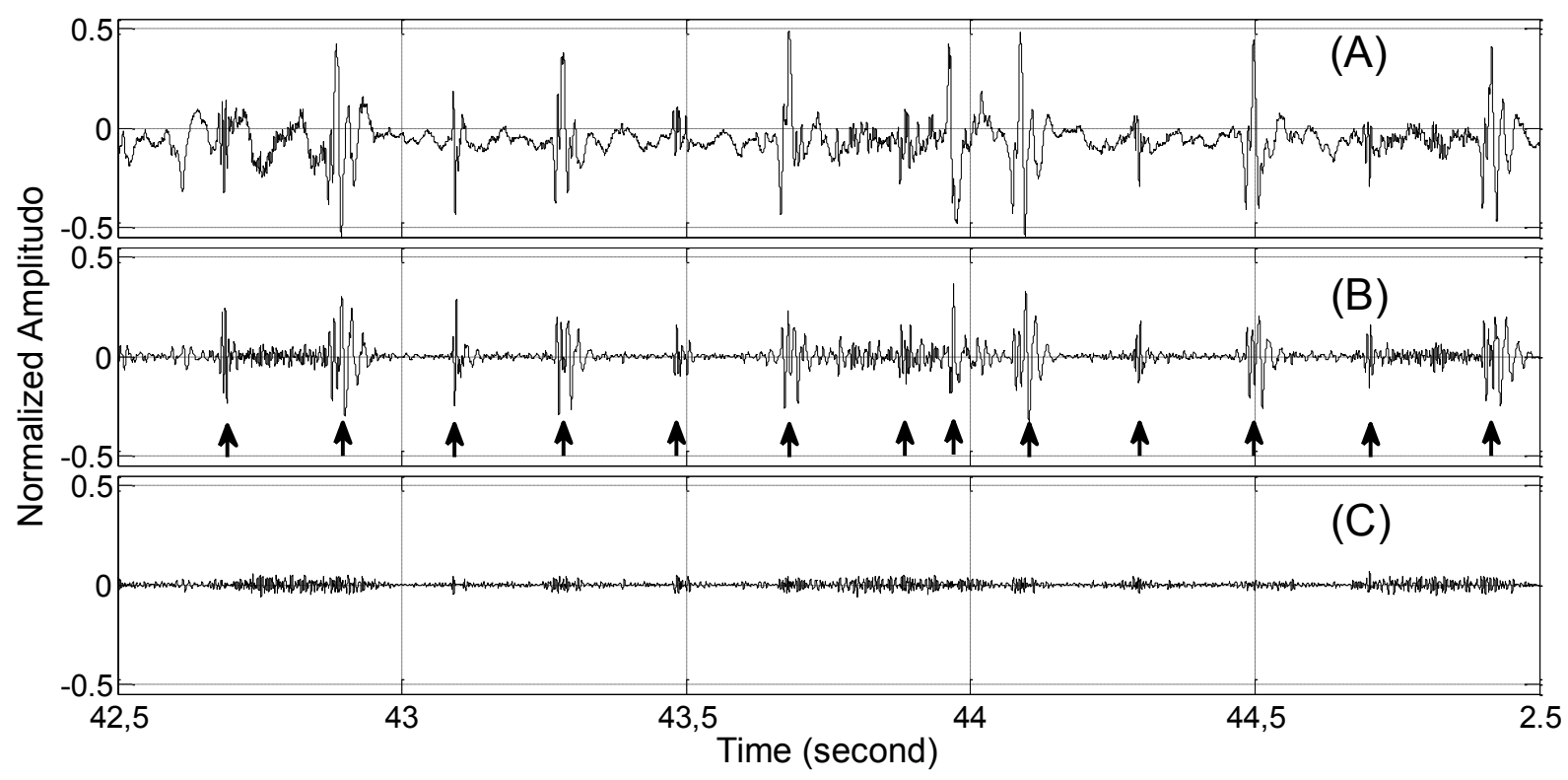

Fig. 5. (A) Breath sound signal from electronic stethoscope (B) Output signal from pre-processing and (C) Output signal from artifacts removal using discrete wavelet transform filter (coift5). Arrow signs show the artifacts in the signal.

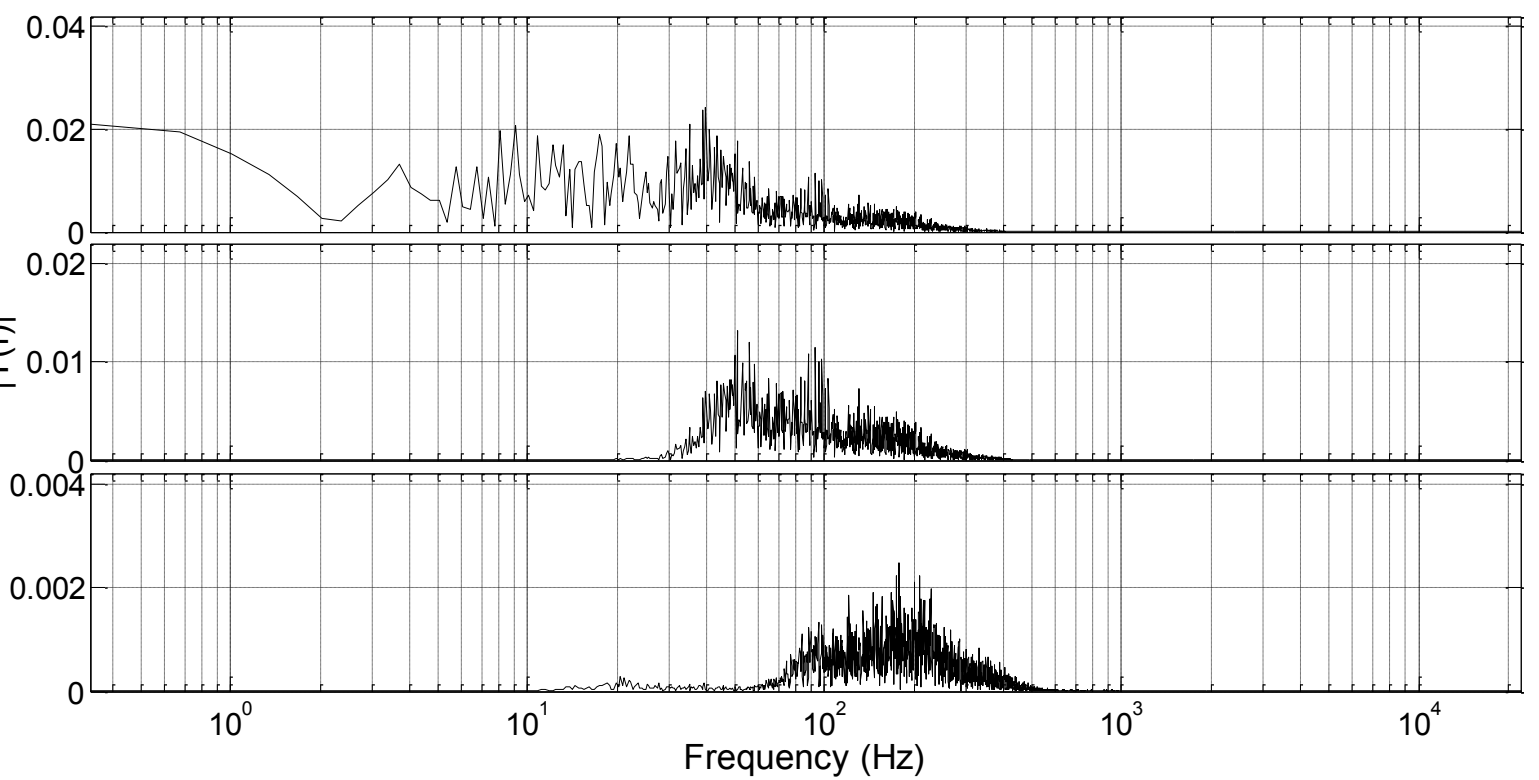

Fig. 6. The spectrum of (A) Breath sound signal from electronic stethoscope (B) Output signal from pre-processing and (C) Output signal from artifacts removal using discrete wavelet transform filter (coift5).

\subsection{Artifacts removal}

In order to reduce the non-stationary artifacts, we implemented a combination of decomposition, thresholding and reconstruction using discrete wavelet transform filter. As the spectrum of the artifacts spread lower than $50 \mathrm{~Hz}$, we designed DWT with ten level decompositions. The artifact components mostly located in the Details coefficients $D_{i}$. Therefore, we attenuated magnitude of the artifacts by comparing with the thresholds given in Table 1. We followed Eq 3 in this process.

For decomposition level 1 to 5 we used threshold value of 10 as artifact signal was minimal in this spectrum.
In spectrum where breath sound and artifacts were overlap, we implemented a moderate threshold $(0.12-$ $0.18)$. Meanwhile, we used lower threshold (0.016 0.023 ) in decomposition level of 9 and 10 to reduce the artifacts. We used the same threshold values for testing Coiflet, Symlet and Daubachies wavelet. The illustration of signal after discrete wavelet transform filter (coift5) is shown in Fig 5(C) whiles its spectrum in Fig 6(C). It can be seen that the artifacts were reduced significantly.

\subsection{Performance of wavelet filter}

In this work, we use three types of wavelet including Coiflet, Symlet and Daubachies. We tested 5, 8, 20 and 32 
orders of wavelet filter. However, for Coiflet we only tested the fifth order as it is its maximum order. Figure 7 illustrates the average of $\triangle \mathrm{SNR}$ from the three types wavelet applied in the nine subjects. The figure shows that 32 orders Symlet (sym32) achieved the highest SNR improvement $(10.65 \mathrm{~dB})$. The coift5 and sym 20 showed relatively similar $\triangle \mathrm{SNR}$ around $10.3 \mathrm{~dB}$. The lowest performance showed in 5 orders symlet (sym5) with average of $\triangle \mathrm{SNR} 9,61 \mathrm{~dB}$. Figure 7 also shows that filters using symmetrical wavelet such as Symlet and Coiflet show better SNR improvement compared to asymmetrical wavelet (Daubechies).

Table 1. Threshold values used in ten decomposition levels of wavelet processing. The term $i$ is the decomposition level, $f_{A}$ is the approximation spectrum, $f_{D}$ is the spectrum of details and $\tau$ is the threshold values.

\begin{tabular}{llll}
\hline $\mathrm{p}$ & $f_{A}(\mathrm{~Hz})$ & $f_{D}(\mathrm{~Hz})$ & $\tau$ \\
\hline \hline 1 & $0-11025$ & $11025-22050$ & 10 \\
2 & $0-5512$ & $5512-11025$ & 10 \\
3 & $0-2756$ & $2756-5512$ & 10 \\
4 & $0-1378$ & $1378-2756$ & 10 \\
5 & $0-689$ & $689-1378$ & 10 \\
6 & $0-344$ & $344-689$ & 0,12 \\
7 & $0-172$ & $172-344$ & 0,14 \\
8 & $0-86$ & $86-172$ & 0,18 \\
9 & $0-43$ & $43-86$ & 0,023 \\
10 & $0-21$ & $21-43$ & 0,016 \\
\hline
\end{tabular}

From the figure, it can be seen that higher order wavelet filters showing better SNR improvement. This occurs as higher order wavelet filters have higher vanishing moment in their wavelet function [9]. Theoretically, if a wavelet filter has more vanishing moment then its scaling function can represent signals that are more complex accurately. This means that it can decompose the artifacts accurately even though the signal was non-stationer. In the same order, the 5 orders Coiflet outperform both Symlet and Daubechies. This occurs as Coif5 has more vanishing moment (10) compared to db5 and sym5 (5).

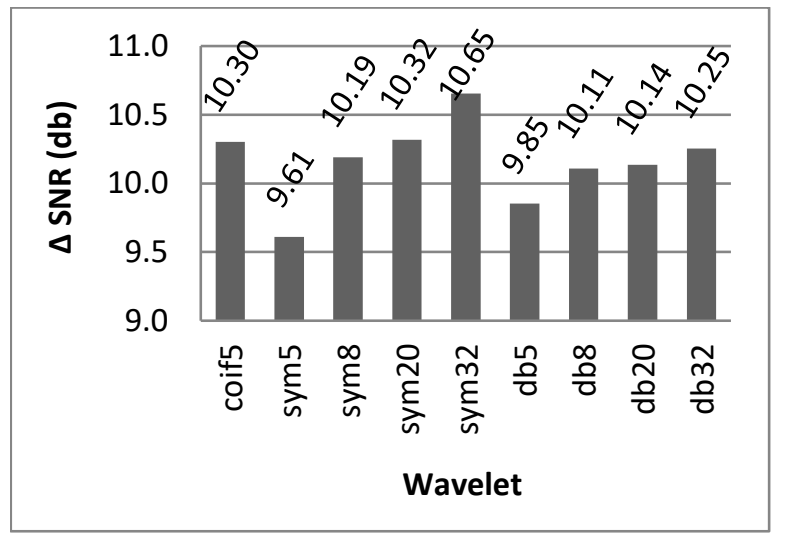

Fig. 7. Signal to noise ratio improvement $(\triangle \mathrm{SNR})$ before and after discrete wavelet transform filter.
Our results show that our developed method was able to remove the unwanted artifacts found in recordings from pediatric subjects. Unlike study in [7], our work does not used breath sound signals from adults. The combination of an electronic stethoscope and a mobile phone for acquiring breath sound is suitable for rural areas. The hardware can be deployed easily. The digital data is also easily saved and transferred for further analysis by other physicians. This method can be used to improve the diagnosis of pneumonia in high prevalence areas such as Nusa Tenggara and Papua [10].

\section{Conclusion}

In this work we address processing complications in breath sound recordings from pediatrics subjects. We employed Thinklab One Digital Stethoscope and a Mobile phone to record the breath sounds. We involved pediatric subject diagnosed as pneumonia. In these subjects, the breath sounds have lower magnitude but in contrast, it has many artifacts due to movements or sounds from other organ such as heart. In real situation, it is difficult to get a reference artifact signal for filtering process to omit the artifacts. Therefore, we proposed to develop a combination of band pass filter with discrete wavelet filter. The results show that our proposed method was able to improve the signal to noise ratio significantly up to $10.655 \mathrm{~dB}$.

\section{References}

1. L. J. Hadjileontiadis, Lung Sounds: An Advanced Signal Processing Perspective. Connecticut: Morgan \& Claypool, 2009.

2. Y. Norisue, Y. Tokuda, M. Koizumi, T. Kishaba, and S. Miyagi, "Phasic characteristics of inspiratory crackles of bacterial and atypical pneumonia," Postgraduate Medical Journal, vol. 84, pp. 432-436, 2008.

3. A. Vyshedskiy, R. M. Alhashem, R. Paciej, M. Ebril, I. Rudman, and J. J. Fredberg, "Mechanism of Inspiratory and Expiratory Crackles," Chest, vol. 135, pp. 156-164, 2009.

4. B. Mathews, S. Shah, R. H. Cleveland, E. Y. Lee, R. G. Bachur, and M. I. Neuman, "Clinical Predictors of Pneumonia Among Children With Wheezing," Pediatrics, vol. 124, pp. e29-e36, 2009.

5. World Health Organization, "IMCI: Integrated Management of Childhood Illnesses," in Management of the child with a serious infection or severe malnutrition: Guidelines for care at the firstreferral level in developing countries, ed: World Health Organization and UNICEF, 2000.

6. A. Chang, "Cough: are children really different to adults?" Cough, vol. 1, p. 7, 2005.

7. L. J. Hadjileontiadis and S. M. Panas, "A waveletbased reduction of heart sound noise from lung sounds," International Journal of Medical Informatics, vol. 52, pp. 183-190, 1998. 
8. O. Rioul and M. Vetterli, "Wavelets and signal processing," IEEE signal processing magazine, vol. 8, pp. 14-38, 1991.

9. H. Cui and G. Song, "Study of the Wavelet Basis Selections," in Computational Intelligence and Security: International Conference, CIS 2006. Guangzhou, China, November 3-6, 2006. Revised Selected Papers, Y. Wang, Y.-m. Cheung, and H. Liu, Eds., ed Berlin, Heidelberg: Springer Berlin Heidelberg, pp. 1009-1017, 2007.

10. Litbang-Kemenkes, "Riset Kesehatan Dasar" vol. 1, B. P. Pengembangan, Ed., ed. Jakarta: Kementrian Kesehatan Republik Indonesia, pp. 67-68, 2013. 